\title{
Piezoresistivity and conductance anisotropy of tunneling-percolating systems
}

\author{
C. Grimaldi ${ }^{1}$, T. Maeder ${ }^{1,2}$, P. Ryser ${ }^{1}$, and S. Strässler ${ }^{1,2}$ \\ ${ }^{1}$ Institut de Production et Robotique, LPM, Ecole Polytechnique Fédérale de Lausanne, CH-1015 Lausanne, Switzerland \\ ${ }^{2}$ Sensile Technologies SA, PSE, CH-1015 Lausanne, Switzerland
}

\begin{abstract}
Percolating networks based on interparticle tunneling conduction are shown to yield a logarithmic divergent piezoresistive response close to the critical point as long as the electrical conductivity becomes nonuniversal. At the same time, the piezoresistivity or, equivalently, the conductivity anisotropy exponent $\lambda$ remains universal also when the conductive exponent is not, suggesting a purely geometric origin of $\lambda$. We obtain these results by an exact solution of the piezoresistive problem on a Bethe lattice and by Monte Carlo calculations and finite-size scaling analysis on square lattices. We discuss our results in relation to the nature of transport for a variety of materials such as carbon-black-polymer composites and $\mathrm{RuO}_{2}$-glass systems which show nonuniversal transport properties and coexistence between tunneling and percolating behaviors.

PACS numbers: 64.60.Fr, 72.20.Fr, 72.60.+g
\end{abstract}

\section{INTRODUCTION}

Tunneling-percolating network models have been suggested to properly describe transport properties of disordered systems in which tunneling coexists with percolation behaviors. Examples of such systems are provided by various carbon-black-polymer composites, 1 for which, close to the metal-insulator transition, the conductivity $\sigma$ follows a percolation-like power law of the form:

$$
\sigma \simeq \sigma_{0}\left(p-p_{c}\right)^{t}
$$

where $\sigma_{0}$ is a prefactor, $p$ is the concentration of the conducting phase, $p_{c}$ is the percolation critical concentration, and $t$ is the critical exponent. $\mathrm{A}$ clear indication that transport is dominated by tunneling between carbon-black grains is prgvided by a large strain and/or volume sensitivity of $\sigma$. Similar situations are encoun tered also in organic conductors-polymer composites, and thick-film resistors made of metal-oxide conductiff grains inhomogeneously embedded in a glassy matrix $\mathbf{6}$. These latter compounds display quite large piezoresistive effects and their use for sensor applications is widespread. 8

Modelling such systems in terms of tunnelingpercolating networks resides on the assumption that the exponential decay $\exp (-2 r / \xi)$ of the tunneling amplitude permits to retain only nearest neighbouring conducting grains. Since the tunneling factor $\xi \propto 1 / \sqrt{V}$ depends only on the potential barrier $V$ separating the grains, a modification of the tunneling distance $r$ induced by an applied strain $\varepsilon$ can lead to a large variation of the total conductivity, and so to large values of the piezoresistive coefficient $d \log \left(\sigma^{-1}\right) / d \varepsilon$. For example both carbonblack-polymer composites and $\mathrm{RuO}_{2}$-based thick-film resistors can display piezoresistive coefficients up to about 30.36

Although percolating network theories predict that the conductivity exponent $t$ should be universal and equal to $t \simeq 2.0$ for three dimensional lattices, 0 tunnelingpercolating systems display important deviations from universality. For example, values of $t$ up to about $t \simeq 6$ have been reported for some kind of carbon-black composites 1 , while $t \simeq 4$ h has been measured for $\mathrm{RuO}_{2}$ based thick-film resistors 10

Nonuniversality has been theoretically proposed to arise from specific conducting-insulating phases distributions such as in the Swiss-cheese model where spherical insulating inclusions are introduced in a continuous conductor.11 An alternative explanation has been put forward specifically for tunneling-percolating materials by arguing that, if the composite has a sufficiently wide distribution of tunneling distances, the distribution of bond conductances between conductive grains has a power-law divergence.12 It is well known that such anomalous distribution can lead to nonuniversality 13

In this paper we argue that the piezoresistive effect, $i$. $e$. , the sensitivity of $\sigma$ upon applied strain, could be a decisive tool to investigate the origin of nonuniversality. We show that if nonuniversality of tunneling-percolating systems is given by a diverging distribution of tunneling conductances, then the piezoresistive response diverges logarithmically at the percolation thresold, while at the same time the piezoresistivity anisotropy remains universal regardless of the universality breakdown of the conductivity exponent $t$. We obtain these results by an analytic solution of the piezoresistive problem on a Bethe lattice and by Monte Carlo calculations and finite-size scaling analysis on square lattices.

This paper is organized as follows. In the next section we introduce the lattice model and the relevant quantities concerning the piezoresistive response. In section III we solve analytically the piezoresistive problem for a Bethe lattice while in Section IV we investigate numerically the square lattice. A discussion of our results in connection with real materials is presented in the last section where also the conclusions are drawn. 


\section{THE MODEL}

Let us consider a random resistor network where the bond conductivity distribution is given by:

$$
\rho(g)=p h(g)+(1-p) \delta(g),
$$

where $p$ is the fraction of bonds with finite conductivity $g$ with distribution $h(g)$. In the following, we implicitly assume that the temperature is high enough to neglect other processes than intergrain tunneling (grain charging effects, Coulomb repulsion). This is certainly a good approximation for thick-film resistors which shows a very weak temperature dependence of $\sigma$ already at room temperature. Hence, within a tunneling-percolation framework, $h(g)$ depends upon the distribution of tunneling distances between nearest neighbouring grains. For narrow distributions, $h(g)$ is peaked around $g=$ $g_{0} \exp (-2 a / \xi)$ where $a$ is the mean intergrain distance, $\xi$ is the tunneling factor, and $g_{0}$ has units of a conductivity. We assume that the main dependence upon the mean intergain distance $a$ is all contained in the argument of the exponential and for simplicity we set $g_{0}=1$. In a first approximation, we can set

$$
h(g)=\delta\left(g-e^{-2 a / \xi}\right),
$$

as an extreme case for narrow distribution for tunneling distances. In this situation, close to the metal-insulator transition, transport follows Eq.(1) with universal exponent $t$ depending only upon dimensionality and all dependence upon tunneling distance $a$ is embodied in the prefactor $\sigma_{0}$ which measures the average bond conductivity. Hence, we expect that a change of the tunneling distance $a$ induced by an applied external strain would affect only $\sigma_{0}$.

Let us consider now the case in which $N$ spherical particles of radius $R_{0}$ are placed at random so that the tunneling distance $r$ between two neighbouring grains fluctuates. When the average distance $a$ between two adjacent grains is such that $a \gg R_{0}$, Balberg argued that the salient feature of the resulting bond conductivity distribution is captured by a power-law divergence at small $g: 12$

$$
h(g)=(1-\alpha) g^{-\alpha},
$$

where $\alpha=1-\xi / 2 a$ and the prefactor $1-\alpha$ assures the correct normalization of $h(g)$ and $\rho(g)$. As first shown by Kogut and Straley, 13 for values of $\alpha$ larger than some dimensionality dependent critical value $\alpha_{c}$, universality breaks down and the transport exponent $t$ becomes $\alpha$ dependent: $t \rightarrow t(\alpha)>t$. The important point here is that since $\alpha$ depends on the mean intergrain distance $a$, then the exponent $t(\alpha)$ can be affected by an applied external strain $\varepsilon$ and this would lead to an anomalous piezoresistive response.

To investigate this issue, let us assume that the network is embedded in a homogeneous elastic medium and that the elastic coefficients of the network and the medium are equal. Moreover we set the bond directions parallel to the axes of a $D$-dimensional cubic lattice. In this situation, if we apply an uniaxial strain $\varepsilon$ along, for example, the $x$-axis, then the tunneling distance for a bond directed along $x$ changes to $a \rightarrow a_{x}=a(1+\varepsilon)$, while the bonds directed along the other orthogonal axes remain unchanged: $a_{i}=a$ for $i \neq x$. The straininduced change of the tunneling distances leads therefore to anisotropic bond conductivity distributions $\rho_{x}(g)$ and $\rho_{i \neq x}(g)=\rho(g)$. For an external electric field $E_{i}$ applied along the $i$-axis and up to linear order in $\varepsilon$, the resulting network conductivities $\sigma_{i}$ are $\sigma_{x}=\sigma\left(1-\Gamma_{\|} \varepsilon\right)$ and $\sigma_{i \neq x}=\sigma\left(1-\Gamma_{\perp} \varepsilon\right)$ where $\sigma$ is the unstrained conductivity and

$$
\Gamma_{\|}=\frac{d \ln \sigma_{x}^{-1}}{d \varepsilon} ; \quad \Gamma_{\perp}=\frac{d \ln \sigma_{i}^{-1}}{d \varepsilon}(i \neq x),
$$

are the longitudinal and transverse piezoresistive coefficients, respectively. These two quantities completely define the piezoresistive properties of the network. For example, the isotropic (or hydrostatic) piezoresistive response $\Gamma$ obtained by applying equal strain $\varepsilon$ along all bonds directions is:14

$$
\Gamma=\frac{d \ln \sigma^{-1}}{d \varepsilon}=\Gamma_{\|}+(Z / 2-1) \Gamma_{\perp},
$$

where $Z$ is the coordination number, while informations about the tortuosity the current has in flowing through the network are given by the piezoresistive anisotropy factor defined as:

$$
\chi=\frac{\Gamma_{\|}-\Gamma_{\perp}}{\Gamma_{\|}},
$$

which measures the degree of macroscopic transport anisotropy. 14 Close to the percolation thresold, $\chi$ displays a power-law of the form:14

$$
\chi \sim\left(p-p_{c}\right)^{\lambda}
$$

where the exponent $\lambda$ is the same of that governing criticality of the conductivity anisotropy $A=1-$ $\sigma_{y} / \sigma_{x}$ of random resistor networks with anisotropic bond conductances.15.16.17 In the present case, in fact, bond anisotropy is induced by the applied uniaxial strain and since $\lambda$ is independent of the degree of bond anisotropy, 18 $A$ and $\chi$ have the same critical behavior with the same exponent $\lambda$.

Having introduced the main quantities defining the piezoresistive properties of random resistor networks, let us now discuss qualitatively the effects of universality breakdown induced by the diverging bond conductances distribution function Eq.(A). The anisotropy factor $\chi$ and its exponent $\lambda$ cannot be accounted in a simple way without explicitly solving the anisotropy problem, which is done in the following sections. However, the hydrostatic piezoresistive response $\Gamma$ can be simply evaluated 
by noticing that, by definition, it is obtained by changing the tunneling distance $a$ to $a(1+\varepsilon)$ for all bond directions. Hence $\Gamma$ can be readily found by differentiating Eq.(1) with respect to $\varepsilon$ :

$$
\Gamma= \begin{cases}\Gamma_{0} & \alpha<\alpha_{c} \\ \Gamma_{0}+(1-\alpha) \ln \left(\frac{1}{p-p_{c}}\right) t^{\prime}(\alpha) & \alpha \geq \alpha_{c}\end{cases}
$$

where $\Gamma_{0}=-d \ln \sigma_{0}^{-1} / d \varepsilon$. For $\alpha<\alpha_{c}$ transport is universal and the piezoresistive response is governed solely by the strain-dependence of $\sigma_{0}$ in Eq.(1) leading to the $p$-independent factor $\Gamma_{0}$. Of course, distribution functions for occupied bonds like Eq.(3) would trivially lead to the same qualitative result, i.e., a piezoresistive response independent of the concentration of occupied bonds. Instead, as soon as transport becomes nonuniversal $\left(\alpha \geq \alpha_{c}\right)$, the tunneling-percolation model of Balberg Eq.(14) predicts a logarithmic divergence of the hydrostatic piezoresistive response. Note that such an anomalous behavior is expected also for distributions more complicated than Eq.(4) as long as their asymptotic behavior for $g \rightarrow 0$ has a power-law divergence with exponent depending upon the tunneling distance.

In the next sections we shall verify the correctness of Eq.(9) and calculate the $p$-dependence of $\chi$ by considering two quite dinstinct cases: the Bethe lattice model, which is paradigmatic of high-dimensionality lattices for which transport is governed by mean-field exponents, 19 and the two-dimensional square lattice.

\section{BETHE LATTICE}

The conductivity problem on a Bethe lattice, or Cayley tree, model has been considered and solved in Refs.[ 20, 21]. As we show below, for this model the concentration dependence of the piezoresistive response in the critical regime can be obtained analytically for both distributions Eqs.(3,4). For simplicity in the following we shall consider a Bethe lattice with coordination number $\left.Z=4\left(p_{c}=1 /(Z-1)=1 / 3\right)\right)$. According to Ref.[ 22 , the distribution $P_{i}(\sigma)$ of conductivities from an arbitrary bond directed along $i=x, y$ to infinity and the current distributions $J_{i}(\sigma)$ induced by an applied electric field $E_{i}$ satisfy the following coupled non-linear integral equations:

$$
\begin{gathered}
P_{x}\left(\sigma_{1}\right)=\int_{0}^{1} d g \rho_{x}(g) \int d \sigma_{2} d \sigma_{3} d \sigma_{4} P_{x}\left(\sigma_{2}\right) P_{y}\left(\sigma_{3}\right) P_{y}\left(\sigma_{4}\right) \\
\quad \times \delta\left[\sigma_{1}-\frac{g\left(\sigma_{2}+\sigma_{3}+\sigma_{4}\right)}{g+\sigma_{2}+\sigma_{3}+\sigma_{4}}\right], \\
J_{x}\left(\sigma_{1}\right)=E_{x} \sigma_{1} P_{x}\left(\sigma_{1}\right)+\int_{0}^{1} d g \rho_{x}(g) \int d \sigma_{2} d \sigma_{3} d \sigma_{4} \\
\frac{g J_{x}\left(\sigma_{2}\right) P_{y}\left(\sigma_{3}\right) P_{y}\left(\sigma_{4}\right)}{g+\sigma_{2}+\sigma_{3}+\sigma_{4}} \delta\left[\sigma_{1}-\frac{g\left(\sigma_{2}+\sigma_{3}+\sigma_{4}\right)}{g+\sigma_{2}+\sigma_{3}+\sigma_{4}}\right] .
\end{gathered}
$$

The corresponding equations for $P_{y}(\sigma)$ and $J_{y}(\sigma)$ are obtained from Eqs.(10,11) by substituting $x$ with $y$ (and vicerversa), and the total conductivities $\sigma_{i}$ are obtained from:

$$
\sigma_{x}=-2 \int_{0}^{\infty} d z J_{x}(z) P_{y}(z) \frac{d\left[P_{x}(z) P_{y}(z)\right]}{d z},
$$

where $P_{i}(z)$ and $J_{i}(z)$ are the Laplace transforms of $P_{i}(\sigma)$ and $J_{i}(\sigma)$, respectively.22

Let us consider first the case of a narrow tunnelingdistances distribution, Eqs.(2,3), for which, in the absence of applied strain, the conductivity is $\sigma=$ $6 \exp (-2 a / \xi)\left(p-p_{c}\right)^{t}$ for $\mid p-p_{q} \ll 1$ where $t=3$ is the universal transport exponent 21 The effect of an applied uniaxial strain, $\varepsilon \neq 0$, can be readily found by following Ref.[ 22]. In the critical region, we find that the piezoresistive coefficients Eq.(5) reduce to:

$$
\Gamma_{\|(\perp)}=\frac{a}{\xi}\left[1+(-) \frac{15}{16}\left(p-p_{c}\right)\right] .
$$

The above result captures the essential physics at the basis of the piezoresistive response of percolating networks. 14 For $p>p_{c}, \Gamma_{\|}>\Gamma_{\perp}$ because the strainsensitivity is stronger for sample conductivities measured along the direction of the applied strain. As $p$ moves towards $p_{c}$, the current currying paths become more and more tortuous decreasing therefore the macroscopic anisotropy induced by $\varepsilon$ until at $p=p_{c}$ the longitudinal and transverse piezoresistive coefficients become equal. 14 From Eq.(13), we find that the piezoresistive anisotropy $\chi$, Eq.(7), goes to zero as:

$$
\chi=\frac{15}{8}\left(p-p_{c}\right)
$$

Hence the anisotropy exponent for the Bethe lattice is $\lambda=1$ in accord with previous results 22

Now, we consider how the piezoresistive response of a Bethe lattice changes when the bond conductances have a power-law distribution as in Eqs.(2,4). In doing so, we generalize the procedure described in Ref. 13 to the anisotropic bond conductance case of Eqs. (10 and perform an expansion in powers of $\varepsilon$. To obtain the piezoresistive coefficients, it is sufficient to keep only terms up to linear order in $\varepsilon$. Here we stress that the resulting piezoresistive response for the Bethe lattice model depends crucially on the sign of $\alpha$. For $\alpha<0$, corresponding to the quite unphysicalrelation $a<\xi / 2$, conductivity is universal with $t=3,13$ and the piezoresistive response can be easily shown to behave qualitatively as in the binary distribution case discussed above (finite values of $\Gamma_{\|(\perp)}$ at $p=p_{c}$ and $\left.\lambda=1\right)$. Instead when $\alpha>0$, the piezoresistive response changes qualitatively as discussed in the following.

From Eq.(10) we find that for $\alpha>0$ the $p-p_{c}$ dependence of the Laplace transforms of $P_{x}(\sigma)$ and $P_{y}(\sigma)$ 


$$
P_{x(y)}(z)=1-\delta f(\omega)+\delta \ln \delta\left[g(\omega)+(-) \delta g_{1}(\omega)\right] \varepsilon,
$$

where $\omega=\delta^{\frac{1}{1-\alpha}} z$ and $\delta=\left(p-p_{c}\right) / p_{c}$. The functions $f(y), g(y)$ and $g_{1}(y)$ satisfy coupled integral equations, but their explicit expressions are not of interest here. By applying the same procedure to Eq.(11) we find:

$J_{x(y)}(z)=\frac{3}{2} \delta^{1+\frac{1}{1-\alpha}} E_{x}\left\{f^{\prime}(\omega)-\ln \delta\left[g^{\prime}(\omega)+(-) \delta g_{2}(\omega)\right] \varepsilon\right\}$.

Finally, by substituting these results into Eq.(12) we obtain

$$
\sigma_{x(y)}=\delta^{3+\frac{\alpha}{1-\alpha}}\left\{a_{0}-a_{1} \varepsilon-\ln \left(\delta^{-1}\right)\left[a_{2}+(-) a_{3} \delta\right] \varepsilon\right\},
$$

where $a_{0}, \ldots, a_{3}$ are positive functions of $\Omega$. For $\varepsilon=0$, we obtain the result of Kogut and Straley: $13 \sigma_{x(y)}=\sigma \sim$ $\left(p-p_{c}\right)^{t(\alpha)}$ with $t(\alpha)=3+\alpha /(1-\alpha)$, indicating that transport is non-universal for $\alpha>\alpha_{c}=0$. In this regime, the piezoresistive coefficients are obtained from the terms of Eq.(17) proportional to $\varepsilon$ :

$$
\Gamma_{\|(\perp)}=\frac{a_{1}+\ln \left(\delta^{-1}\right)\left[a_{2}+(-) a_{3} \delta\right]}{a_{0}} \sim \ln \left(\frac{1}{p-p_{c}}\right) .
$$

We have arrived therefore at the result that as long as the tunneling-distance distribution is such that transport becomes non-universal, then the piezoresistive response diverges logarithmically as $p \rightarrow p_{c}$. This must be contrasted with the finite value of $\Gamma_{\|}$and $\Gamma_{\perp}$ at $p=p_{c}$, Eq.(13), obtained from the simple binary distribution Eqs.(2):33). From Eq.(18) we obtain also that the piezoresistive anisotropy $\chi$, Eq.(17), reduces to

$$
\chi=\frac{2 a_{3}}{a_{2}} \delta \propto\left(p-p_{c}\right) .
$$

Hence $\chi$ goes to zero with universal exponent $\lambda=1$ irrespectively of the logarithmic divergence of $\Gamma_{\|(\perp)}$ and of the universality breakdown of $\sigma$.

\section{SQUARE LATTICE}

The results of the last section have been derived for a Bethe lattice and are therefore in general relevant for high-dimensionality $(D \geq 6)$ lattices 19 To investigate the piezoresistive response for low dimensional networks, we consider now a square lattice. We employ the transfermatrix method of Derrida and Vannimenus which permits to calculate exactly the conductivity of a longstrip of width $N$ (along $x$ ) and length $L \gg N$ (along $y$ ). 23 The distribution of the $N \times L$ conductors follows Eq.(2, (1) and the longitudinal and transverse piezoresistive coefficients are obtained by setting $a_{x}=a(1+\varepsilon), a_{y}=a$ and $a_{x}=a$, $a_{y}=a(1+\varepsilon)$, respectively, with $\varepsilon=0.001$. For each value of $\alpha>0$ at the percolation thresold $p=p_{c}=1 / 2$,

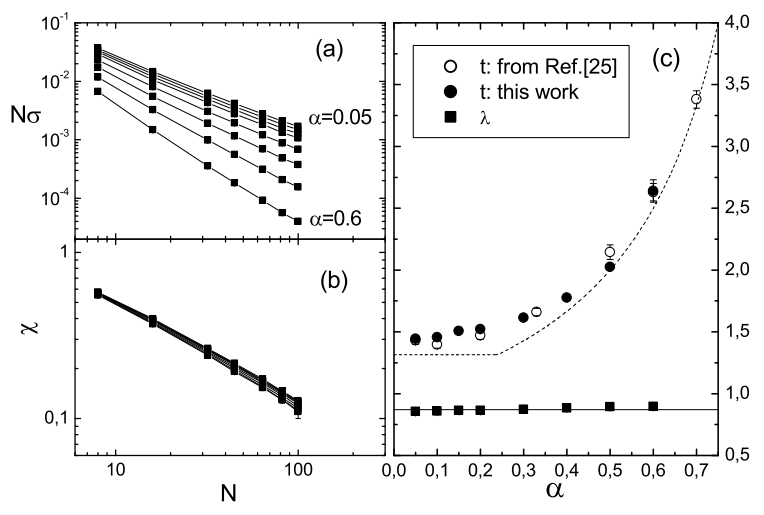

FIG. 1. Unstrained conductivity $\sigma$ (a) and piezoresistive anisotropy $\chi(\mathrm{b})$ calculated at $p=p_{c}=1 / 2$ as function of the width $N$ of the strip for $\alpha=0.05,0.1,0.15,0.2,0.3, \ldots 0.6$. (c): solid circles: conductivity exponent $t$ as a function of the distribution exponent $\alpha$. Empty circles: results from Ref. 25. Dashed curve is the theoretical estimate $t(\alpha)=1.3158$ for $\alpha<\alpha_{c}=0.24$ and $t(\alpha)=1 /(1-\alpha)$ for $\alpha>\alpha_{c}$. Solid squares: conductivity (or piezoresistivity) anisotropy exponent $\lambda$. Solid line: average value $\lambda=0.87$.

we perform the calculations at $N=8\left(L=1 \times 10^{8}\right)$, $N=16\left(L=7.5 \times 10^{7}\right), N=32\left(L=2.5 \times 10^{7}\right)$, $N=45\left(L=2 \times 10^{7}\right), N=64\left(L=1 \times 10^{7}\right), N=$ $82\left(L=1 \times 10^{7}\right)$, and $N=100\left(L=0.8 \times 10^{7}\right)$.

In Fig. 11 and Fig. 2 we show our results for the unstrained conductivity $\sigma$, the longitudinal and transverse piezoresistive coefficients $\Gamma_{\|(\perp)}$, and the piezoresistive anisotropy $\chi$. From the $\sigma$-vs- $N$ data of Fig.11(a) we extract the conductivity exponent $t$ from the finite-size scaling relation $\sigma(N)=c_{1} N^{-t / \nu}\left(1+c_{2} / N\right)$ where $\nu=\frac{4}{3}$ is the correlation length exponent 24 The resulting $t$ values (solid circles) are reported in Fig.11(c) as function of the power-law distribution exponent $\alpha$ of Eq.(何). For comparison, we report also the $t$ values obtained in Ref. 25 by a different numerical method (empty circles). As expected, for $\alpha$ sufficiently large, the $t$-exponent increases well beyond its universal value $t \simeq 1.3,24,25$ signalling breakdown of universality. According to Refs. 25, 26, universality is lost for $\alpha>\alpha_{c}=0.24 \pm 0.08$, and the conductivity exponent should follow the relation $t(\alpha)=1 /(1-\alpha)$ reported in Fig.11(c) by the dashed curve. Our numerical results, as those of Ref. 25, agree well with the theoretical expectations for large values of $\alpha$ but somehow overestimates the $t$-values for $\alpha \sim \alpha_{c}$. This deviation from the theoretical expectations should be due to finite-size effects amplified by the presence of two competing fixed points.25.26

In contrast to the large $\alpha$-dependence of $\sigma(N)$, the data for different $\alpha$ values of the piezoresistive anisotropy $\chi$ reported in Fig.1 (b) collapse all in a single curve. Hence, the piezoresistivity (or conductivity) anisotropy exponent $\lambda$ [filled squares in Fig. [1 (c)] estimated from the 


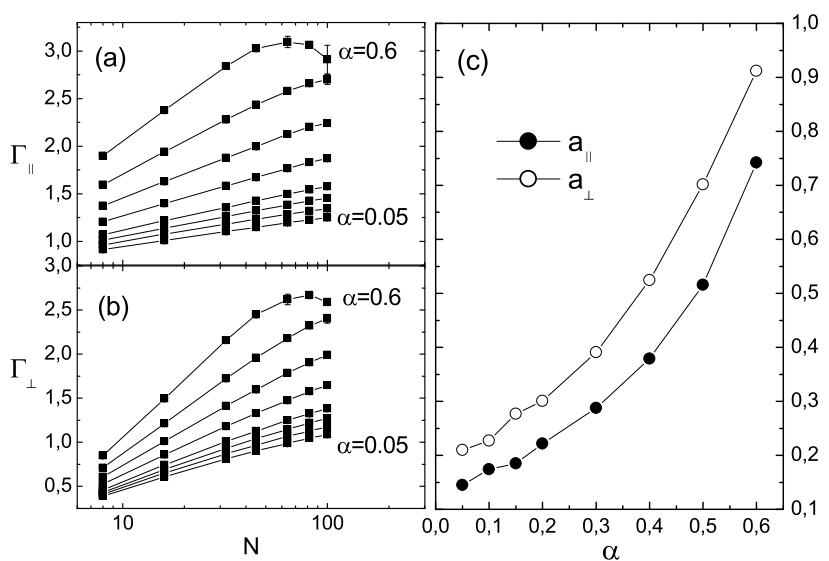

FIG. 2. (a) and (b): longitudinal and transverse piezoresistive coefficients as a function of $N$ for different values of the power-law distribution exponent $\alpha$. (c): prefactor of $\Gamma_{\|(\perp)}=a_{\|(\perp)} \ln (N)$ as a function of $\alpha$.

finite-size scaling relation $\chi(N)=c_{3} N^{-\lambda / \nu}\left(1+c_{4} / N\right)$ does not show appreciable variations over the entire range of $\alpha$-values considered.27 This is in striking contrast with $\lambda=t-\beta_{B}$, where $\beta_{B} \simeq 0.48$ is the fraction of conducting bones in the backbone, conjectured to hold true for $D=2$ in Ref. 17. As for the Bethe lattice case, the universality of $\lambda$ also for $\alpha>\alpha_{c}$ suggests that this exponent depends only upon the geometry of the conducting cluster and is not influenced by $t$.

The results for the piezoresistive coefficients $\Gamma_{\|}$and $\Gamma_{\perp}$ as functions of $N$ are reported in Fig.2(a) and Fig.2(b), respectively. With the exclusion of the large $N$ values for $\alpha=0.6$, which we think are affected by too small values of $L, \Gamma_{\|}$and $\Gamma_{\perp}$ follow approximatively a $\ln (N)$ behavior. This signals a $\ln \left[\left(p-p_{c}\right)^{-1}\right]$ divergence of the piezoresistive coefficients as $p \rightarrow p_{c}$. How the logarithmic divergence depends upon $\alpha$ is studied in Fig.2(c) where we plot the prefactors $a_{\|}$and $a_{\perp}$ of the finite-size scaling law: $\Gamma_{\|(\perp)}=a_{\|(\perp)} \ln (N)(1+\cdots)$. The correction-toscaling terms $\cdots$ which best fitted the data where proportional to $1 / N$ and to $1 / \ln (N)$. Clearly, $a_{\|(\perp)}$ is a monotonous increasing function of $\alpha$ indicating that the logarithmic divergence of $\Gamma_{\|(\perp)}$ is stronger for $\alpha$ larger. In analogy with the results on the Bethe lattice, we would expect that $a_{\|(\perp)}$ vanishes for $\alpha<\alpha_{c}$, so that $\Gamma_{\|(\perp)}$ has a finite limit at $p=p_{c}$. However the $a_{\|(\perp)}$ data of Fig.2(2) are small but nonzero even for $\alpha<\alpha_{c}=0.24$. We think that this is due to the same finite-size errors affecting the unstrained conductivity exponent, Fig.11(c), which lead to a spurious $\alpha$-dependence of $\sigma$ and consequently to $a_{\|(\perp)} \neq 0$.

\section{DISCUSSION AND CONCLUSIONS}

Let us discuss now the applicability of our theory to real materials. The thermal expansion effect on the resistance of nonuniversal $(t \simeq 3.0)$ carbon-black-polymer

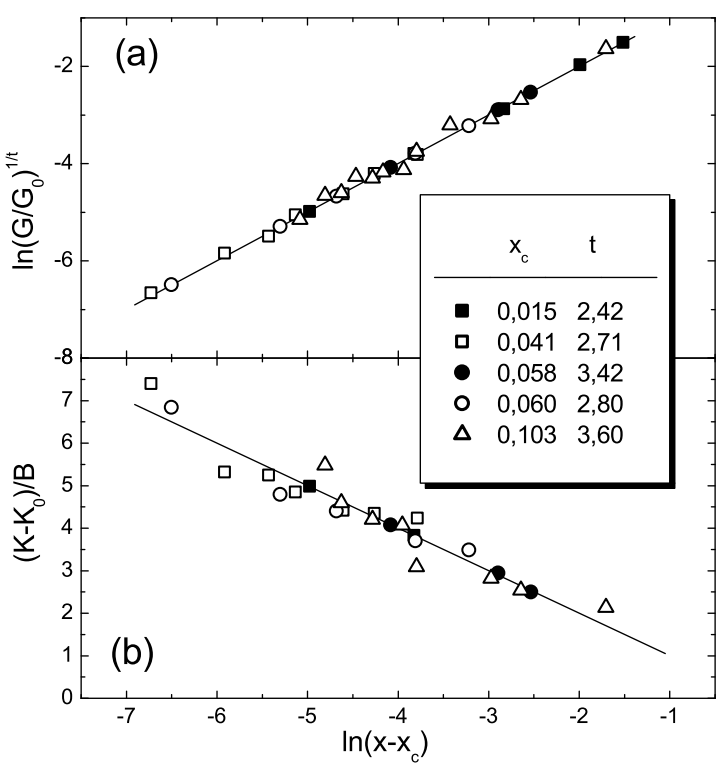

FIG. 3. Conductance $G$ (a) and piezoresistance $K$ (b) of $\mathrm{RuO}_{2}$-glass composites for various $\mathrm{RuO}_{2}$ volume concentrations $x$. Open symbols: Ref. 6. Solid symbols: Ref. 月.

composite reported in Ref. 1 provides a first indirect clue. From the resistance- $v s$-volume data we have extracted a hydrostatic piezoresistive coefficient $\Gamma$ of about 30 which keeps increasing as the volume is expanded by the temperature. If volume expansion effectively reduces the carbon-black concentration, we obtain a piezoresistive coefficient enhancement as the concentration $p$ moves down to its critical value. However, due to the uncontrolled effect of polymer melting on the microstructure of the composite, we have been unable to single out any logarithmic divergence of the piezoresistivity response.

A much clearer situation is found for $\mathrm{RuO}_{2}$-based thick-film resistors. In Fig.3(a) we report conductance $G$ measurements on several $\mathrm{RuO}_{2}$-glass composites for different values of the metal volume concentration $x$ 6. When $\ln \left(G / G_{0}\right)^{1 / t}$ is plotted as a function of $\ln \left(x-x_{c}\right)$, where $G_{0}$ is a prefactor and $x_{c}$ is the critical volume concentration, the whole set of data collapses into a single straight line indicating a power law of the form:

$$
G \simeq G_{0}\left(x-x_{c}\right)^{t} .
$$

In Fig.3(a), the different symbols refer to different relative particles sizes of the glass and $\mathrm{RuO}_{2}$ and the values of $x_{c}$ and $t$ which best fit the experimental data are reported in the inset. Clearly, all composites display values of $t$ much higher than the three dimensional universal value $t \simeq 2.09$

The origin of such universality breakdown is investigated in Fig.3(b) where we plot the $x$-dependence of the longitudinal piezoresistance coefficient $K \overline{\bar{B}} d \ln G^{-1} / d \varepsilon$ obtained by cantilever bar measurements. 6 目 The mea- 
sured $K$ values fit reasonably well with a logarithmically divergent function of the form:

$$
K \simeq K_{0}+B \ln \left(\frac{1}{x-x_{c}}\right)
$$

represented in the $\left(K-K_{0}\right) / B$-vs- $\ln \left(x-x_{c}\right)$ plot of Fig.3(b) by the straight line. Since all the samples lie well within the critical region [Fig.3(a)], we expect that the piezoresistive anisotropy $\chi$ is sufficiently small to regard $K$ as a good approximation of the isotropic (hydrostatic) piezoresistance coefficient.28 Hence, the data of Fig. 3 are fully consistent with Eq.(9) for $\alpha \geq \alpha_{c}$ and provide a rather good example of the effect we have described in this paper.

Unfortunately we are not aware of reported measurements recording $\chi$ as a function of $x$, so that the universality of $\lambda$ claimed here even for $\alpha \geq \alpha_{c}$ cannot be verified. However the measurements reported in of Ref. 29 show that $\chi$ decreases as the sheet resistance of commercial $\mathrm{RuO}_{2}$-based thick-film resistors increases. This is in qualitative accord with $\chi \sim\left(x-x_{c}\right)^{\lambda}$ if higher resistance values are due to lower $\mathrm{RuO}_{2}$ concentrations.

In summary, we have shown by means of analytical and numerical results that when the tunneling exponent $\alpha=1-\xi / 2 a$ of the power-law distribution Eq.(位) is such that transport becomes non-universal, the piezoresistive response changes drastically leading to a logarithmic divergence of the piezoresistive coefficients as $p \rightarrow p_{c}$. In addition, we have demonstrated that despite of the universality breakdown of transport, the conductivity anisotropy exponent $\lambda$ remains universal. These features seem to be quite robust and calls for experimental verifications on systems like carbon-black-polymer composites and thick-film resistors for which tunneling-percolation mechanism of transport have been proposed and nonuniversality has been reported. imental results on carbon-black-polymer composites, and especially $\mathrm{RuO}_{2}$ thick-film resistors 9.0 seem to indicate that indeed these systems are in the diverging tunneling conductance distribution regime.

${ }^{1}$ I. Balberg, Carbon 40, 139 (2002).

2 Z. Rubin, S. A. Sunshine, M. B. Heaney, I. Bloom, and I. Balberg, Phys. Rev. B 59, 12196 (1999).

${ }^{3}$ E. K. Sichel, P. Sheng, J. I. Gittleman, and S. Bozowski, Phys. Rev. B 24, 6131 (1981).

${ }^{4}$ M. B. Heaney, Appl. Phys. Lett. 69, 2602 (1996).

${ }^{5}$ S. Paschen, M. N. Bussac, L. Zuppiroli, E. Minder, and B. Hilti, J. Appl. Phys. 78, 3230 (1995).

${ }^{6}$ P. F. Carcia, A. Suna, and W. D. Childers, J. Appl. Phys. 546002 (1983).

${ }^{7}$ M. Tamborin, S. Piccinini, M. Prudenziati, and B. Morten, Sensor and Actuators A 58, 159 (1997).
${ }^{8}$ M. Prudenziati, Handbook of Sensors and Actuators (Elsevier, Amsterdam, 1994), p.189.

9 J. P. Clerc, V. A. Podolskiy, and A. K. Sarichev, Eur. Phys. J. B 15, 507 (2000).

10 A. Kusy, Physica B 240, 226 (1997).

${ }^{11}$ B. I. Halperin, S. Feng, and P. N. Sen, Phys. Rev. Lett. 54, 2391 (1985); S. Feng, B. I. Halperin, and P. N. Sen, Phys. Rev. B 35, 197 (1987).

${ }^{12}$ I. Balberg, Phys. Rev. Lett. 59, 1305 (1987).

${ }^{13}$ P. M. Kogut and J. Straley, J. Phys. C 12, 2151 (1979).

${ }^{14}$ C. Grimaldi, P. Ryser, and S. Strässler, Phys. Rev. B 64, 064201 (2001); ibid. J. Appl. Phys. 92, 1981 (2002).

15 B. I. Shklovskii, Phys. Status Solidi (b) 85, K111 (1978).

16 A. K. Sarychev and A. P. Vinogradoff, J. Phys. C: Solid State Phys. 12, L681 (1979); ibid. 16, L1073 (1983).

17 S. Mukhopadhyay and M. Sahimi, J. Stat. Phys. 74, 1301 (1994).

${ }^{18}$ C. J. Lobb, D. J. Frank, and M. Tinkham, Phys. Rev. B 23, 2262 (1981).

${ }^{19}$ D. Stauffer and A. Aharony, Introduction to Percolation Theory (Taylor \& Francis, London, 1992).

${ }^{20}$ R. B. Stinchcombe, J. Phys. C 7, 179 (1974).

21 J. P. Straley, J. Phys. C 10, 3009 (1977).

22 J. P. Straley, J. Phys. C: Solid St. Phys. 13, 4335 (1980).

${ }^{23}$ B. Derrida and J. Vannimenus, J. Phys. A: Math. Gen. 15, L557 (1982).

24 J. G. Zabolitzky, Phys. Rev. B 30, R4077 (1984).

${ }^{25}$ M. Octavio and C. J. Lobb, Phys. Rev. B 43, 8233 (1991).

${ }^{26}$ J. Machta, R. A. Guyer, and S. M. Moore, Phys. Rev. B 33, 4818 (1986).

27 The average value of $\lambda$ over the different values of $\alpha$ is $\lambda=0.87 \pm 0.02$ which is only somewhat larger than $\lambda=$ $0.819 \pm 0.011$ estimated in Ref. 17 .

28 The piezoresistance $(K)$ and the piezoresistive $(\Gamma)$ coefficients differ by an additive constant which depends only upon the geometry of the sample 14

${ }^{29}$ M. Hrovat, J. Holc, D. Belavič, and S. Šoba, J. Mater. Sci. Lett. 13, 992 (1994). 